\title{
Sub-ppm Laser Spectroscopy of Antiprotonic Helium and a CPT-Violation Limit on the Antiprotonic Charge and Mass
}

\author{
M. Hori, ${ }^{1}$ J. Eades, ${ }^{1}$ R. S. Hayano, ${ }^{2}$ T. Ishikawa, ${ }^{2}$ J. Sakaguchi, ${ }^{2}$ E. Widmann, ${ }^{2}$ H. Yamaguchi, ${ }^{2}$ H. A. Torii, ${ }^{3}$ B. Juhász ${ }^{4}$ \\ D. Horváth, ${ }^{5}$ and T. Yamazaki ${ }^{6}$ \\ ${ }^{1}$ CERN, CH-1211 Geneva 23, Switzerland \\ ${ }^{2}$ Department of Physics, University of Tokyo, 7-3-1 Hongo, Bunkyo-ku, Tokyo 113-0033, Japan \\ ${ }^{3}$ Institute of Physics, University of Tokyo, Komaba, Meguro-ku, Tokyo 153-8902, Japan \\ ${ }^{4}$ Institute of Nuclear Research of the Hungarian Academy of Sciences, H-4001 Debrecen, Hungary \\ ${ }^{5}$ KFKI Research Institute for Particle and Nuclear Physics, H-1525 Budapest, Hungary \\ ${ }^{6}$ RI Beam Science Laboratory, RIKEN, Wako, Saitama 351-0198, Japan
}

(Received 28 March 2001; published 9 August 2001)

\begin{abstract}
Six laser-resonant transitions have been detected in metastable antiprotonic helium atoms produced at the CERN Antiproton Decelerator. They include UV transitions from the last metastable states in the $v=n-\ell-1=0$ and 1 cascades. Zero-density frequencies were obtained from measured pressure shifts with fractional precisions between $1.3 \times 10^{-7}$ and $1.6 \times 10^{-6}$. By comparing these with QED calculations and the antiproton cyclotron frequency, we deduce that the antiproton and proton charges and masses agree to within $6 \times 10^{-8}$ with a confidence level of $90 \%$.
\end{abstract}

DOI: 10.1103/PhysRevLett.87.093401

PACS numbers: $36.10 .-\mathrm{k}, 14.20 . \mathrm{Dh}, 32.70 . J z$

We here describe measurements of six transition frequencies in metastable antiprotonic helium atoms $\left(\bar{p} \mathrm{He}^{+} \equiv e^{-}-\bar{p}-\mathrm{He}^{2+}\right)[1,2]$, and compare them with values obtained from three-body QED calculations. Two transitions were in the previously unstudied UV region; two others were to final states with large Auger widths which provide particularly stringent tests of such calculations. These studies were done using the Antiproton Decelerator (AD) recently built at CERN.

A variety of antiprotonic atoms exists for every element, but only $\bar{p} \mathrm{He}^{+}$atoms have microsecond-scale lifetimes against annihilation. This extreme longevity (typically $\tau=3-4 \mu \mathrm{s})$ arises because antiprotons in states with large principal $(n \sim 38)$ and angular momentum $(\ell \sim n)$ quantum numbers (see Fig. 1) cannot easily deexcite by Auger emission of the electron. With this electron in place, the atom is protected against collisional Stark mixing with low- $\ell$ states, which overlap with the nucleus. It deexcites by radiating a series of optical-frequency photons, as the antiproton traverses a constant $v=n-\ell-1$ cascade of these metastable states.

Recent variational calculations [3-5] claim a precision of $<1 \times 10^{-7}$, for radiative transition frequencies involving states with small natural widths $(\Gamma \leq 50 \mathrm{MHz})$. Relativistic [4] and one-loop QED corrections [5] are taken into account, as well as nuclear size and order- $\alpha^{4}$ effects. Another calculation using the nonadiabatic coupledrearrangement-channel method [6] has also been made. The two calculations agree within $\leq 2 \times 10^{-7}$ for transitions with natural widths of $\Gamma<50 \mathrm{MHz}$, while a larger difference is seen $\left(>2 \times 10^{-6}\right)$ for states having large Auger widths $(\Gamma \geq 15 \mathrm{GHz})$.

As in previous experiments $[7,8]$, we measured the delayed annihilation time spectrum (the distribution of the number of annihilations, as a function of the time elapsed since $\bar{p} \mathrm{He}^{+}$formation). We tuned pulsed laser beams to stimulate antiproton transitions from metastable states to levels that are not protected in the way described above, thereby revealing the resonance condition between the laser beam and the atom as a sharp peak in the annihilation rate (see Fig. 2). We have already measured one such transition frequency of $(n, \ell)=(39,35) \rightarrow(38,34)$ to an accuracy of $5 \times 10^{-7}$, which agreed with theoretical calculations at a level of $2 \times 10^{-6}$ [9].

The present experiments were done during the first months of operation at the $\mathrm{AD}$, which provided a pulsed beam containing $2 \times 10^{7}$ antiprotons, with an energy of $5.3 \mathrm{MeV}$, a pulse length of $250 \mathrm{~ns}$, and a repetition rate of 1 pulse per 2 min. Metastable $\bar{p} \mathrm{He}^{+}$atoms were produced by stopping the antiproton pulses in a cryogenic helium

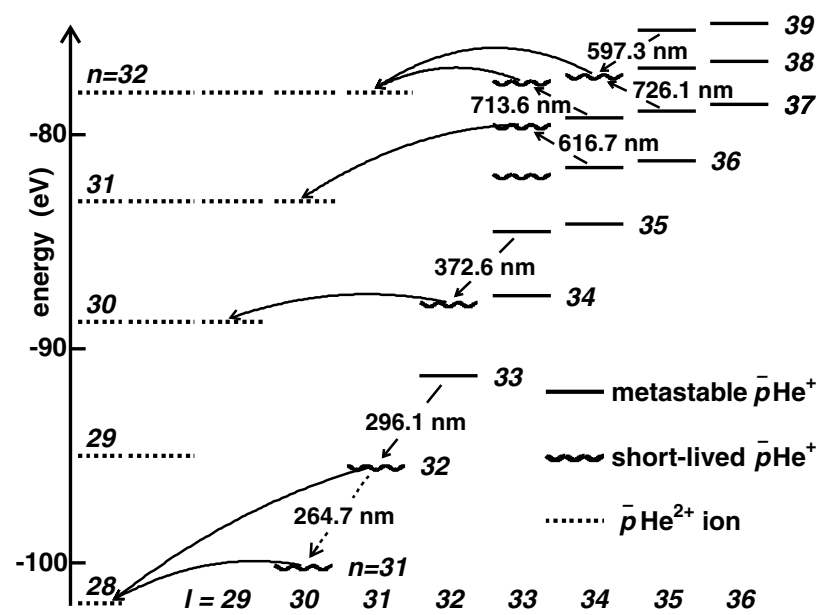

FIG. 1. Portion of the energy level diagram of antiprotonic helium. Curved arrows indicate Auger transitions with minimum $|\Delta \ell|$. Positions of seven transitions are shown, but the one at $\lambda=264.7 \mathrm{~nm}$ was not observed (see text). 


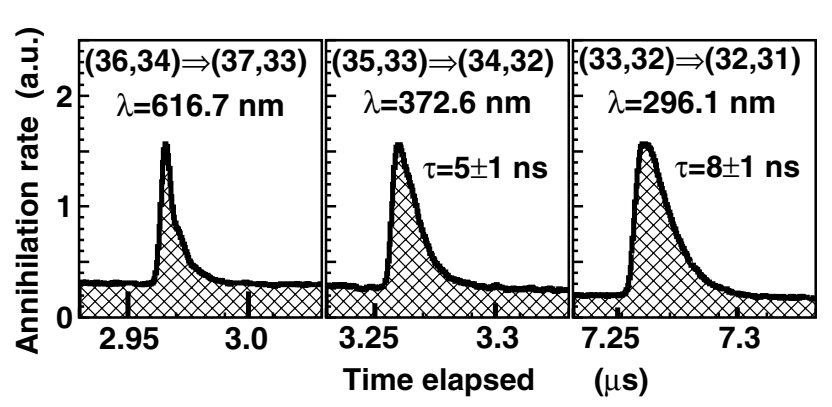

FIG. 2. Laser-induced annihilation peaks, at laser pulse timings producing the highest peak intensities.

gas target (see Fig. 3), at temperatures $T=5.5 \pm 0.2 \mathrm{~K}$ and pressures from $P=0.2$ to 2.5 bars. The spatial profile of this pulse was measured nondestructively by a parallel plate secondary emission chamber, placed near the entrance of the target. Although most antiprotons annihilated promptly after stopping in the helium, 3\% of them formed some $5 \times 10^{5}$ metastable atoms which proceeded to annihilate with an average lifetime of $\tau=3-4 \mu \mathrm{s}$. Charged particles emerging from all these annihilations produced Cherenkov light in two 2-cm-thick plates of UV-transparent Lucite, which covered a solid angle of $2 \pi$ steradians seen from the target. The envelope of the resulting light pulse thus consisted of a bright, 250-ns-long flash from the promptly annihilating antiprotons, followed by a much longer but less intense tail from delayed annihilations of the metastable atoms. Laserinduced peaks (see Fig. 2) were superimposed on this tail. The light pulse was detected by a fine-mesh photomultiplier (Hamamatsu Photonics R5505GX-ASSYII) developed for this experiment, in which the electron multiplication could be gated off during the initial light flash. The output pulse therefore included only delayed annihilations, and had an amplitude of $0.2-0.3 \mathrm{~V}$ and a length of $15 \mu \mathrm{s}$ or more. It was recorded using a digital oscilloscope with a bandwidth of $1 \mathrm{GHz}$.

A dye laser (Lambda Physik Scanmate 2E) pumped by the second or third harmonic $(\lambda=532 / 355 \mathrm{~nm})$ of a $Q$-switched $\mathrm{Nd}$ :YAG laser (Coherent Infinity) was used to generate laser pulses with a wavelength of $\lambda=529-745 \mathrm{~nm}$, a pulse length of 4-6 ns, a diameter

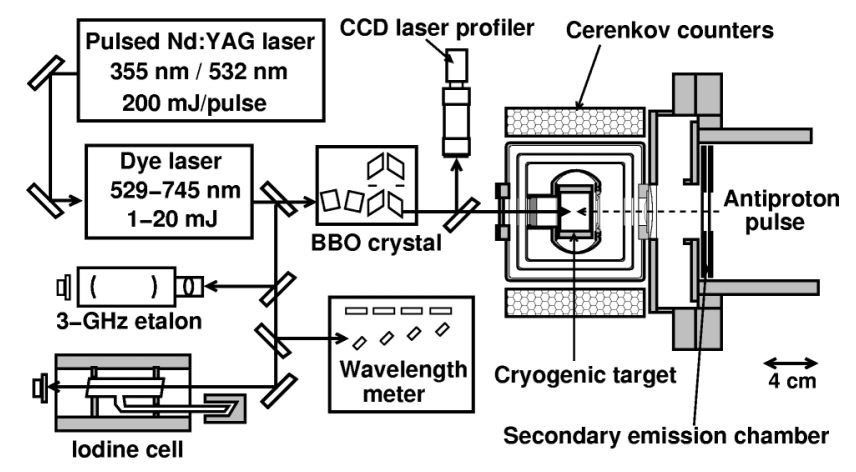

FIG. 3. Experimental setup (for details see text). of $1 \mathrm{~cm}$, and a power density of $0.1-1.0 \mathrm{~mJ} / \mathrm{cm}^{2}$. The metastable atoms could be irradiated at any time $t=$ $0.2-15 \mu \mathrm{s}$ after their birth (see Fig. 2) by adjusting the triggering timing of the Nd:YAG laser. This laser, being equipped with a seeded, relay-imaging, phaseconjugate mirror cavity, could be fired with a timing jitter of less than $0.5 \mathrm{~ns}$ and an intensity fluctuation of less than $2 \%$. An intracavity etalon was installed in the oscillator of the dye laser, optimized for minimum bandwidth (typically $0.6 \mathrm{GHz}$ ) at wavelengths around $730 \mathrm{~nm}$. To produce the UV laser pulses needed to induce the resonances $(35,33) \rightarrow(34,32)$ at $372.6 \mathrm{~nm},(33,32) \rightarrow(32,31)$ at $296.1 \mathrm{~nm}$, and $(32,31) \rightarrow(31,30)$ at $264.7 \mathrm{~nm}$, the output of the dye laser was frequency doubled in a BBO crystal.

The spatial profile of the laser pulse was measured using a charge-coupled (CCD) camera with a fiberoptic plate, the time profile with a UV-sensitive, highbandwidth $(f=1 \mathrm{GHz}) p-i-n$ photodiode, and the intensity with a pyroelectric detector. The wavelength was measured by a wavelength meter (ATOS Lambdameter), consisting of four Fizeau interferometers embedded in a thermostabilized monolithic quartz block filled with neon gas, each interferometer being read out by a CCD. The highest-resolution interferometer had a free-spectral range of $3.8 \mathrm{GHz}$ and a resolution of $0.6 \mathrm{GHz}$. The line profile of each pulse was recorded, and pulses whose bandwidth exceeded $0.6 \mathrm{GHz}$ (typically half of them) were rejected. The stability of the instrument was checked over a 24-h period using a cw frequency-stabilized, single-line mode helium-neon laser, and found to be better than $\pm 30 \mathrm{MHz}$. The wavelength was also measured independently using a temperature-stabilized etalon having a free spectral range of $3 \mathrm{GHz}$. During the two-minute intervals between the arrival of antiproton pulses, the laser wavelength was calibrated against rovibrational absorption lines of molecular iodine [10-12].

The profiles of the six transitions are shown in Fig. 4 (left), each point including data collected from four antiproton pulses. The resonances were typically $0.8-2.0 \mathrm{GHz}$ wide, including contributions from the 0.3-0.8 GHz Doppler broadening, the laser bandwidth, and the hyperfine splitting caused by the coupling between the orbital angular momentum of the antiproton and the electronic spin. The splitting was largest for the unfavored $(\Delta n=1, \Delta \ell=-1)$ transition $(37,35) \rightarrow(38,34)$ at $\lambda=726.1 \mathrm{~nm}[13]$, the resonance profile (see Fig. 4) of which shows two distinct peaks separated by an interval of $\Delta_{\mathrm{HFS}}=1.8 \pm 0.1 \mathrm{GHz}$. Theoretical calculations [14] show that the hyperfine splitting is much smaller $\left(\Delta_{\mathrm{HFS}} \leq 0.5 \mathrm{GHz}\right)$ for the favored $(\Delta n=-1, \Delta \ell=-1)$ transitions. The resonance centroids were obtained by fitting the profiles with two Voigt functions, superimposed on each other and separated by the theoretical value of the hyperfine splitting.

As the atomic density of the target was increased between $\rho=(0.1-3) \times 10^{21} \mathrm{~cm}^{-3}$, the transition frequencies $\nu$ of the known resonances $(39,35) \rightarrow(38,34)$ 

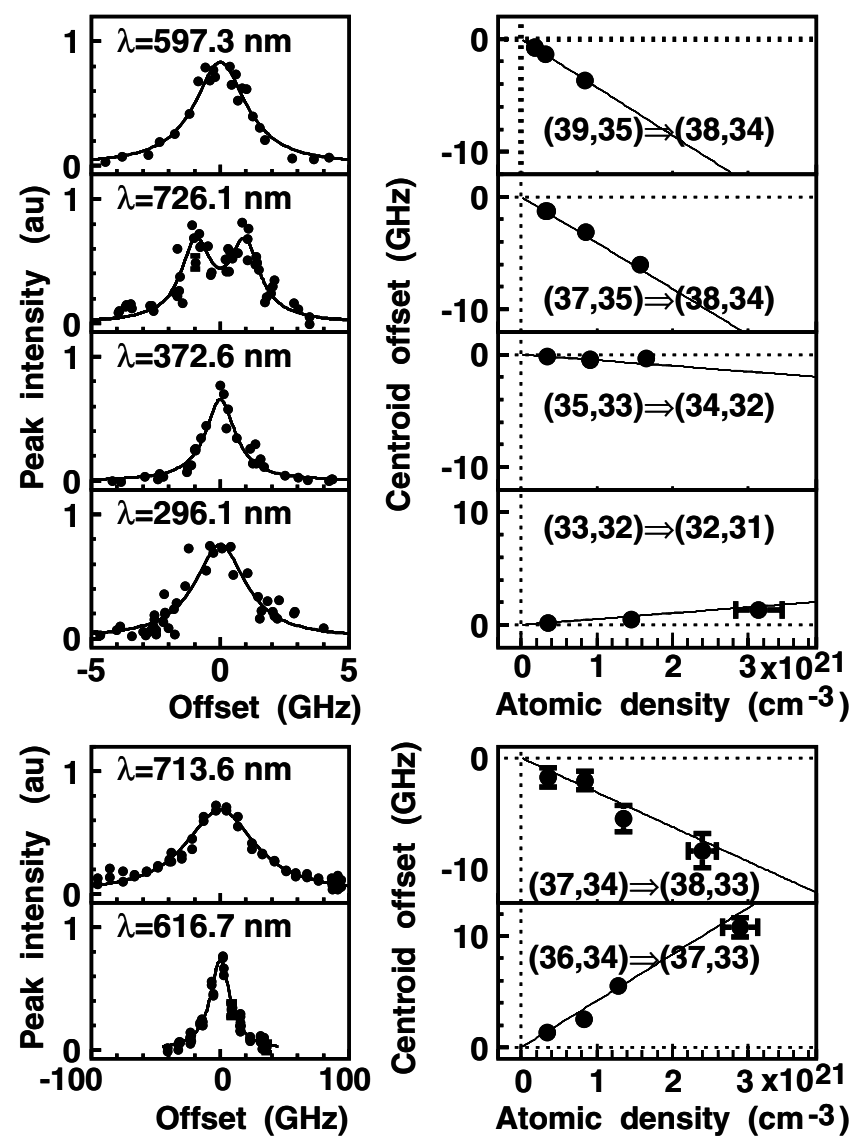

FIG. 4. Resonance profiles of antiprotonic helium (left), transition frequencies at various target densities (right).

at a wavelength of $\lambda=597.3 \mathrm{~nm}[7],(37,35) \rightarrow(38,34)$ at $726.1 \mathrm{~nm} \quad[8,13]$, and $\quad(37,34) \rightarrow(38,33)$ at $713.6 \mathrm{~nm}$ [8] were found to redshift with a gradient of $d \nu / d \rho=-3 \times 10^{-21}$ to $-4 \times 10^{-21} \mathrm{GHz} \cdot \mathrm{cm}^{3}$ (see Fig. 4, right). The experimental values all lie within $\pm 20 \%$ of the results of recent quantum chemistry calculations [15] (see Table I).

Past experiments were made on states having principal quantum numbers of $n=37-39$. To reach the lowerlying states, we searched for and detected the transition $(36,34) \rightarrow(37,33)$ at $616.7 \mathrm{~nm}$ (see Fig. 2). The resonance was found to have a large linewidth of $\Gamma=15 \pm$ $4 \mathrm{GHz}$ (see Fig. 4), corresponding to the resonance daugh- ter state having a lifetime of $\tau=11 \pm 3$ ps. A recent calculation [16] predicted that such a short lifetime is caused by a strong coupling to an electronically excited $\bar{p} \mathrm{He}^{+}$state, where the electron occupies the $3 d$ orbital, and the antiproton the state $(32,31)$. The transition frequency was found to blueshift with a gradient of $d \nu / d \rho=$ $(3.8 \pm 0.5) \times 10^{-21} \mathrm{GHz} \cdot \mathrm{cm}^{3}$ as the target density was increased, which disagrees with theoretical calculations (see Table I) that were made assuming only a single state of $1 s$ electron configuration.

According to Auger calculations, the states $(35,33)$ and $(32,31)$ are the lowest-lying metastable states in the $v=n-\ell-1=1$ and $v=0$ cascades, respectively (see Fig. 1). To observe these states as parent states of laser resonances, the UV transitions $(35,33) \rightarrow(34,32)$ at $\lambda=372.6 \mathrm{~nm}$ and $(32,31) \rightarrow(31,30)$ at $264.7 \mathrm{~nm}$ were searched for. The former transition was detected as expected (see Fig. 2), the decay profile of the resonance spike [17] indicating an Auger lifetime of $\tau=5 \pm 1 \mathrm{~ns}$ for the resonance daughter state $(34,32)$. However, the latter transition was not observed, which we suspected was due to the resonance parent state $(32,31)$ being short lived. To examine this possibility, we searched for the transition $(33,32) \rightarrow(32,31)$ at $296.1 \mathrm{~nm}$, and a strong resonance spike was indeed detected (see Fig. 2). The decay profile indicates that the state $(32,31)$ has an unexpectedly short lifetime of $\tau=8 \pm 1 \mathrm{~ns}$, the reason for which is not understood. The 372.6-nm and 296.1-nm transitions were found to have relatively small frequency shifts of $d \nu / d \rho=-(0.4 \pm 0.1) \times 10^{-21}$ and $(0.4 \pm 0.1) \times$ $10^{-21} \mathrm{GHz} \cdot \mathrm{cm}^{3}$, respectively (see Fig. 4 and Table I), which agree with theoretical calculations.

We obtained the zero-density frequencies by linear extrapolation of the experimental data (see Table I and Fig. 5). The four transitions with natural widths of $\Gamma<50 \mathrm{MHz}$ agree with the theoretical values within $\left|\nu_{\text {th }}-\nu_{\text {exp }}\right| / \nu_{\text {exp }} \leq 5 \times 10^{-7}$, the experimental errors $(80-150 \mathrm{MHz})$ being of the same order as the QED one-loop vacuum polarization effect [4]. The experimental accuracy was limited by the statistical error (20-40 MHz), and the systematic errors of calibrating the laser frequency using Doppler-broadened iodine lines (70-140 MHz), and of extrapolating the frequencies to zero density $(20-50 \mathrm{MHz})$. The best agreements $\left(<1.3 \times 10^{-7}\right.$ and $<1.5 \times 10^{-7}$ ) were found for the two UV transitions

TABLE I. Zero density values of the resonant wavelengths, transition frequencies, and their density shifts: experimental results and theoretical calculations.

\begin{tabular}{|c|c|c|c|c|c|c|}
\hline \multirow{2}{*}{$\begin{array}{c}\text { Transition } \\
(n, \ell) \rightarrow\left(n^{\prime}, \ell^{\prime}\right)\end{array}$} & \multirow{2}{*}{$\begin{array}{l}\text { Wavelength (nm) } \\
\text { Expt. }\end{array}$} & \multicolumn{3}{|c|}{ Transition frequency $(\mathrm{GHz})$} & \multicolumn{2}{|c|}{ Shift $\left(\times 10^{21} \mathrm{GHz} \cdot \mathrm{cm}^{3}\right)$} \\
\hline & & Expt. & Korobov [5] & Kino [6] & Expt. & Bakalov [15] \\
\hline$(39,35) \rightarrow(38,34)$ & $597.25680(12)$ & $501949.01(10)$ & 501948.746 & 501948.828 & $-4.2(2)$ & -3.97 \\
\hline$(37,34) \rightarrow(38,33)$ & $713.5844(12)$ & $420121.9(7)$ & 420123.2 & 420121.53 & $-3.3(8)$ & -3.58 \\
\hline$(36,34) \rightarrow(37,33)$ & $616.7266(6)$ & $486102.7(5)$ & 486142.0 & 486104.43 & $3.8(5)$ & -1.90 \\
\hline$(35,33) \rightarrow(34,32)$ & $372.58280(5)$ & $804633.11(10)$ & 804633.086 & 804633.127 & $-0.4(1)$ & -0.21 \\
\hline
\end{tabular}




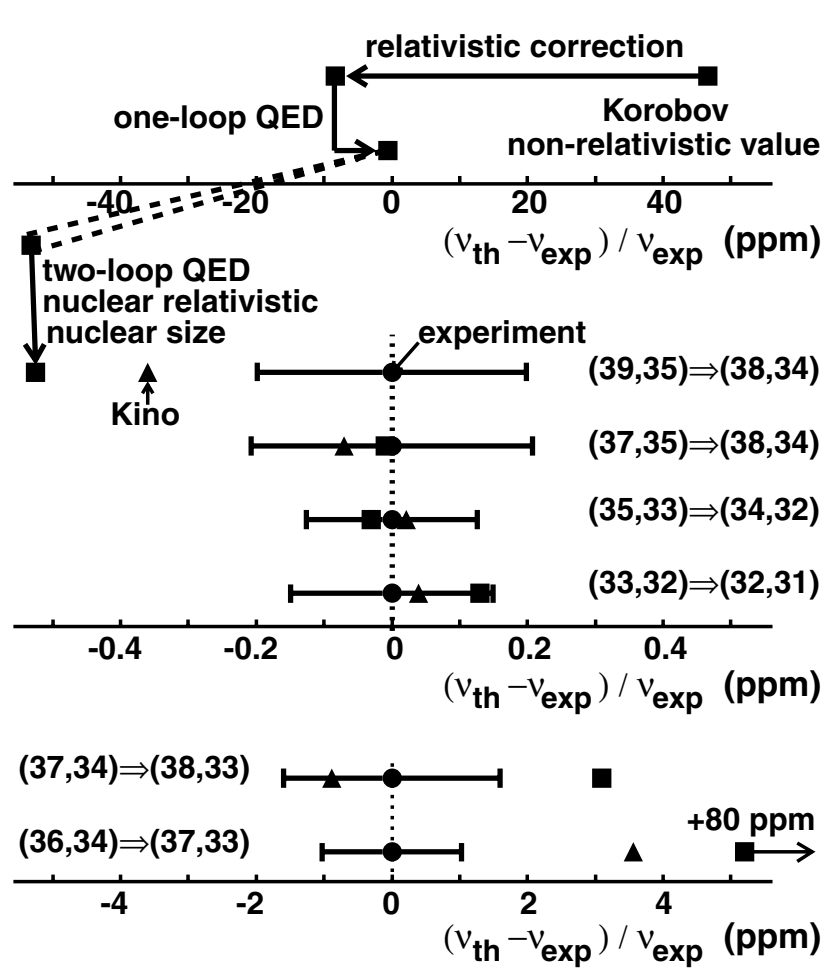

FIG. 5. Comparisons between experimental (filled circles with errors) and theoretical (squares [5] and triangles [6]) values for six transition frequencies in $\bar{p} \mathrm{He}^{+}$atoms. Note the factor of 10 and 100 differences in the axis scales.

$(35,33) \rightarrow(34,32)$ at $372.6 \mathrm{~nm}$ and $(33,32) \rightarrow(32,31)$ at $296.1 \mathrm{~nm}$, which showed small frequency shifts. The largest difference between experiment and theory among the four was for the transition $(39,35) \rightarrow(38,34)$ at $597.3 \mathrm{~nm}$, but the reason for this is not known.

The differences were much larger $\left(\geq 1 \times 10^{-6}\right)$ for the two transitions $(37,34) \rightarrow(38,33)$ at $713.6 \mathrm{~nm}$ and $(36,34) \rightarrow(37,33)$ at $616.7 \mathrm{~nm}$, which have linewidths of $\Gamma \geq 15 \mathrm{GHz}$. For the latter transition, the discrepancy between experiment and the variational calculation [5] was particularly large $\left(8 \times 10^{-5}\right)$ due to the coupling of the state $(37,33)$ to a broad, electronically excited $\bar{p} \mathrm{He}^{+}$state, while the coupled-rearrangement-channel calculation [6] had a better agreement of $<4 \times 10^{-6}$. On account of their large experimental errors, these transitions have been excluded in the following analysis.

By comparing the measured frequencies with the theoretical values for the four transitions with natural widths of $\Gamma<50 \mathrm{MHz}$, we can set a limit on the possible difference between the antiprotonic charge $\left(Q_{\bar{p}}\right)$ and mass $\left(M_{\bar{p}}\right)$, and the values for the proton $\left(Q_{p}\right.$ and $\left.M_{p}\right)$. Two independent observables are needed for such derivations; we used the transition frequencies measured in the present work which have a scale of the antiprotonic Rydberg constant $\left(R_{\bar{p}} \propto M_{\bar{p}} Q_{\bar{p}}^{2}\right)$, and the cyclotron frequency $\left(\omega_{\bar{p}} \propto\right.$ $\left.Q_{\bar{p}} B / M_{\bar{p}}\right)$ measured in Penning trap experiments to a pre- cision of $9 \times 10^{-11}$ [18]. Limits on the charge and mass were deduced $[6,9]$ from the equation,

$$
\delta=\frac{Q_{p}+Q_{\bar{p}}}{Q_{p}} \sim \frac{M_{p}-M_{\bar{p}}}{M_{p}}=\frac{1}{f} \frac{\nu_{\text {th }}-\nu_{\text {exp }}}{\nu_{\exp }} .
$$

Values of 2.5-6.1 for $f$ were estimated by noting that when Kino et al. [6] changed the values of the antiprotonic charge and mass by $1 \mathrm{ppm}$ (under the stringent constraint of Ref. [18] on $\omega_{\bar{p}}$ ), the transition energies changed by $2.5-6.1 \mathrm{ppm}$ depending on the transition. A value of $(-2.5 \pm 2.3) \times 10^{-8}$ was found for $\delta$, by comparing our four measured frequencies with the two sets of theoretical values, and averaging over the experimental errors. This shows that the antiprotonic charge (and mass) is in agreement with that of the proton to a precision of $6 \times 10^{-8}$ at a confidence level of $90 \%$, which is 300 times more stringent than values obtained from x-ray spectroscopy of antiprotonic atoms $[19,20]$, and a factor of 8 improvement over our past experiments $[9,19]$.

We thank the CERN AD team and cryogenic laboratory for their help, and V. I. Korobov, Y. Kino, and D. Bakalov for communicating theoretical results prior to publication. This work was supported by the Grant-in-Aid for Creative Basic Research (10NP0101) of Monbukagakusho of Japan, the Hungarian Scientific Research Fund (OTKA T033079 and TeT-Jap-4/98), and the Japan Society for the Promotion of Science.

[1] M. Iwasaki et al., Phys. Rev. Lett. 67, 1246 (1991).

[2] T. Yamazaki et al., Nature (London) 361, 238 (1993).

[3] V. I. Korobov, Phys. Rev. A 54, R1749 (1996).

[4] V. I. Korobov and D. D. Bakalov, Phys. Rev. Lett. 79, 3379 (1997).

[5] V. I. Korobov, Hyperfine Interact. 119, 185 (1999); (private communication).

[6] Y. Kino, M. Kamimura, and H. Kudo, Hyperfine Interact. 119, 201 (1999); (private communication).

[7] N. Morita et al., Phys. Rev. Lett. 72, 1180 (1994).

[8] T. Yamazaki et al., Phys. Rev. A 55, R3295 (1997).

[9] H. A. Torii et al., Phys. Rev. A 59, 223 (1999).

[10] I. Velchev et al., J. Mol. Spectrosc. 187, 21 (1998).

[11] S. C. Xu et al., J. Mol. Spectrosc. 201, 256 (2000).

[12] B. Bodermann et al., Eur. Phys. J. D 11, 213 (2000).

[13] E. Widmann et al., Phys. Lett. B 404, 15 (1997).

[14] D. Bakalov and V.I. Korobov, Phys. Rev. A 57, 1662 (1998).

[15] D. Bakalov et al., Phys. Rev. Lett. 84, 2350 (2000); (private communication).

[16] O. I. Kartavtsev, D. E. Monakhov, and S. I. Fedotov, Phys. Rev. A 61, 062507 (2000); 63, 019901(E) (2001).

[17] M. Hori et al., Phys. Rev. A 58, 1612 (1998).

[18] G. Gabrielse et al., Phys. Rev. Lett. 82, 3198 (1999).

[19] Particle Data Group, D. E. Groom et al., Eur. Phys. J. C 15, 1 (2000).

[20] R. J. Hughes and B. I. Deutch, Phys. Rev. Lett. 69, 578 (1992). 